\title{
Consensus Mutagenesis and Ancestral Reconstruction Provide Insight into the Substrate Specificity and Evolution of the Front-End $\Delta 6$-Desaturase Family
}

Dongdi Li, ${ }^{\infty}$ Adam M. Damry, ${ }^{\infty}$ James R. Petrie, Thomas Vanhercke, Surinder P. Singh, and Colin J. Jackson*

Cite This: Biochemistry 2020, 59, 1398-1409

Read Online

ACCESS | Lلll Metrics \& More | 回 Article Recommendations ｜ sl Supporting Information

ABSTRACT: Marine algae are a major source of $\omega$-3 long-chain polyunsaturated fatty acids ( $\omega 3$-LCPUFAs), which are conditionally essential nutrients in humans and a target for industrial production. The biosynthesis of these molecules in marine algae requires the desaturation of fatty acids by $\Delta 6$-desaturases, and enzymes from different species display a range of specificities toward $\omega 3$ - and $\omega 6$-LCPUFA precursors. In the absence of a molecular structure, the structural basis for the variable substrate specificity of $\Delta 6$-desaturases is poorly understood. Here we have conducted a consensus mutagenesis and ancestral protein reconstruction-based analysis of the $\Delta 6$-desaturase family, focusing

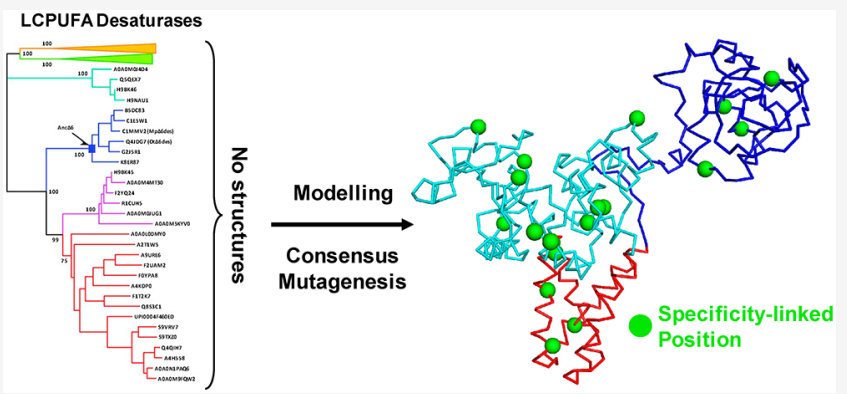
on the $\omega 3$-specific $\Delta 6$-desaturase from Micromonas pusilla ( $\mathrm{Mp} \Delta 6 \mathrm{des})$ and the bispecific $(\omega 3 / \omega 6) \Delta 6$-desaturase from Ostreococcus tauri (Ot $\Delta 6 \mathrm{des}$ ). Our characterization of consensus amino acid substitutions in $\mathrm{Mp} \Delta 6 \mathrm{des}$ revealed that residues in diverse regions of the protein, such as the $\mathrm{N}$-terminal cytochrome $b_{5}$ domain, can make important contributions to determining substrate specificity. Ancestral protein reconstruction also suggests that some extant $\Delta 6$-desaturases, such as Ot $\Delta 6$ des, could have adapted to different environmental conditions by losing specificity for $\omega 3$-LCPUFAs. This data set provides a map of regions within $\Delta 6$-desaturases that contribute to substrate specificity and could facilitate future attempts to engineer these proteins for use in biotechnology.

$\omega-3$ long-chain polyunsaturated fatty acids ( $\omega 3$-LCPUFAs) such as docohexaenoic acid (DHA, C22:6 $6^{\Delta 4,7,10,13,16,19}$ ) and eicosapentaenoic acid (EPA, C20:5 $5^{\Delta 5,8,11,14,17}$ ) are conditionally essential fatty acids in humans that are vital to healthy metabolism. ${ }^{1}$ Deficiencies in these dietary fatty acids result in a reduction of cell membrane fluidity that can adversely affect neurotransmission and contribute to cardiovascular disease., ${ }^{2,3}$ However, most animals have a limited capacity to synthesize $\omega 3$-LCPUFAs as they lack key desaturase enzymes that catalyze the production of $\omega 3$-LCPUFAs from precursors such as linoleic acid (LA, C18:2 $2^{\Delta 9,12}$ ) and $\alpha$-linolenic acid (ALA, C18:3 $3^{\Delta 9,12,15}$ ) by introducing double bonds into fatty acid acyl chains. In nature, marine algae are some of the primary producers of $\omega 3$-LCPUFAs via the $\Delta 6$-desaturasedependent pathway, which then accumulate in fish oils that serve as a major dietary source of $\omega 3$-LCPUFAs for humans. However, due to the scarcity of marine resources, relying wholly on fish oils for dietary $\omega 3$-LCPUFA requirements is projected to be unsustainable. More efficient methods of $\omega 3$ LCPUFA production are thus of interest, the development of which requires a better understanding of the enzymes that catalyze the rate-limiting commitment step in their synthesis, $\Delta$ 6-desaturases. ${ }^{4-7}$
$\Delta 6$-Desaturases belong to the family of membrane-bound front-end desaturases, which catalyze double bond formation at specific positions near the fatty acid carboxyl end. $\Delta 6$ Desaturases specifically introduce a double bond between the acyl-chain C6 and C7 and preferentially target substrates such as LA and ALA that already possess several double bonds, including bonds at the $\Delta 9$ position. ${ }^{4,8}$ Although crucial to LCPUFA production pathways, these enzymes have proven to be extremely difficult to characterize in depth. Very few highresolution structures of membrane-bound fatty acid desaturases have been determined, none of which are of $\Delta 6$ desaturases, and these enzymes cannot be easily purified in an active form. Sequence-based modeling and topology studies have nonetheless provided cursory information about the organization of these enzymes, generally predicting a fourtransmembrane helix $(\mathrm{TMH})$ topology that resembles that of

Received: February 8, 2020

Revised: $\quad$ March 23, 2020

Published: March 25, 2020 
the few crystallized $\Delta$ 9-desaturases. ${ }^{9-11}$ These TMHs form one portion of the enzymes' desaturase domain, which also contains the enzyme active site and three conserved histidinerich motifs (His boxes) that are thought to coordinate a di-iron center. $^{12-15} \Delta 6$-Desaturases are also known to require a fused $b_{5}$ cytochrome domain at their $\mathrm{N}$-terminus for their activity, which functions as an electron donor during catalysis. ${ }^{16-18}$ However, although several other desaturases are capable of accepting electrons from cytoplasmic cytochromes, mutating key residues in the $b_{5}$ domain of $\Delta 6$-desaturases renders the enzymes nonfunctional, hinting that this domain may serve additional roles in $\Delta 6$-desaturases. ${ }^{19,20}$

The LCPUFA $\Delta 6$-desaturase-dependent biosynthesis pathway was recently reconstructed in model and crop plant systems using the Micromonas pusilla $\Delta 6$-desaturase (Mp $\Delta 6$ des) (Supplementary Figure 1). ${ }^{4,5,21,22} \mathrm{Mp} \Delta 6$ des was chosen to catalyze this reaction, because it is among the most active $\Delta 6$-desaturases that preferentially recognize $\omega 3$ - over $\omega 6$-LCPUFA precursors to be identified from algae or plants. ${ }^{4,6}$ Other $\Delta 6$-desaturases, such as that from Ostreococcus tauri (Ot $\Delta 6 \mathrm{des}$ ), do not display a preference for $\omega 3$-LCPUFA precursors and are therefore less desirable for biotechnology applications. $^{23,24}$ Although $\mathrm{Mp} \Delta 6 \mathrm{des}$ is an efficient and moderately $\omega 3$-specific $\Delta 6$-desaturase, further optimization of this enzyme, particularly in terms of its specificity for $\omega 3$ substrates such as $\alpha$-linolenic acid, would result in enhanced recombinant LCPUFA production in plants. Mutagenesis studies have revealed that the second putative $\mathrm{TMH}$, the regions adjacent to the conserved histidine motifs, and the $\mathrm{C}$ terminus affect the regioselectivity and the preferred substrate chain length of membrane desaturases..$^{10,11,25,26}$ However, although $\Delta 6$-desaturases have been identified that naturally prefer either $\omega 3$ - or $\omega 6$-fatty acids, ${ }^{23,27-31}$ detailed mutagenesis studies to modify $\omega 3 / \omega 6$-fatty acid preference have not been performed, to the best of our knowledge. Similarly, the evolution of $\omega 3 / \omega 6$-fatty acid specificity, including divergence between $\mathrm{Mp} \Delta 6 \mathrm{des}$ and $\mathrm{Ot} \Delta 6 \mathrm{des}$, has not been investigated in detail.

Here, we have conducted an intensive mutagenesis study aimed at understanding $\Delta 6$-desaturase specificity through the introduction of consensus mutations from the larger algal desaturase family and ancestral protein reconstruction. This study identifies several regions of the protein that affect substrate preference, including a more significant role for the cytochrome $b_{5}$ domain than previously thought. These results combined with modeling and topology predictions also suggest mechanisms by which desaturases gate substrate specificity. Overall, this work takes important steps to elucidate the complexities of desaturase substrate specificity, laying a foundation for the future engineering of these critical enzymes along the $\omega 3$-LCPUFA production pathway.

\section{METHODS}

Yeast Vectors and Strains. An isogenic strain of Saccharomyces cerevisiae S288C (his3D200, ura3-52, leu2D1, lys2D202 and trpD63) ${ }^{32}$ was used throughout this project for the expression of the wild type and variants of $M p \Delta 6 \mathrm{des}$. The pYES2-ura yeast expression vector containing the wild-type $\mathrm{Mp} \Delta 6 \mathrm{des}$ gene was used for the expression and mutagenesis of the desaturases. Escherichia coli TOP10 cells were used for plasmid preparation and cloning procedures.

Phylogenetic Analysis and Structural Modeling of Algal $\Delta 6$-Desaturases. Mp $\Delta 6$ des (UniProt C1MMV2) was used as the query sequence, and BLASTP was used as the search engine to retrieve 100 homologues of front-end desaturases in the nonredundant protein sequence database. $^{33,34}$ After the sequence set was processed using the CD-HIT suite ${ }^{35}$ to remove sequences with sequences that are $>90 \%$ identical, duplicate sequences and highly similar sequences were removed. The number of sequences was reduced to 57, which were aligned with the MUSCLE algorithm $^{36}$ in the MEGA6 program. ${ }^{37}$

A phylogenetic tree was constructed using the maximumlikelihood method implemented by the MEGA6 program. ${ }^{37}$ The phylogenetic tree was constructed using the maximumlikelihood method with the LG rate matrix and bootstrapped with 100 replicates. The ancestral protein $A n c \Delta 6$ was constructed from the phylogenetic tree using the empirical Bayesian method that is implemented in the PAML4 software package. ${ }^{38}$ Because of the low degree of sequence conservation of the N-terminus, fragment $1-54$ of wild-type $\mathrm{Mp} \Delta 6 \mathrm{des}$ replaced the low-confidence inferred $\mathrm{N}$-terminus of the ancestor.

The transmembrane topology prediction was carried out using the CCTOP Web server ${ }^{39}$ and the visual representation generated using the PROTTER Web server. ${ }^{40}$ Amino acid sequences for $\mathrm{Mp} \Delta 6 \mathrm{des}$, Ot $\Delta 6 \mathrm{des}$, and Anc $\Delta 6$ were then submitted to the Phyre2 Web server for homology protein structure prediction. ${ }^{41}$ Desaturase domains for all three were modeled using the two available acyl-CoA desaturase structures [Protein Data Bank (PDB) entries $4 \mathrm{YMK}^{42}$ and $\left.4 \mathrm{ZYO}^{43}\right]$ as templates, and cytochrome $b_{5}$ domains were modeled using several free cytochrome $b_{5}$ structures as templates.

Construction of Mp $\mathbf{\Delta} 6$ des Variants. The previously constructed pYES2-ura plasmid with the Mp $\Delta 6$ des gene cloned between the KpnI and SacI sites was used as the template to generate the desired single mutants. Primer pairs were designed according to the QuikChange site-directed mutagenesis protocol. ${ }^{44}$ A T7 primer was used with the antisense primer, and a Gall terminator primer was used with the sense primer of each mutagenesis primer pair to generate DNA fragments with the desired mutation in the overlapping region. The DNA fragments and the KpnI/SacI-digested pYES2 vector were assembled using an in-house Gibson assembly kit. ${ }^{45}$ The resulting coding regions were confirmed by sequencing. The 23 algal $\Delta 6$ consensus mutants were constructed and cloned by GenScript.

Functional Expression in Yeast. Yeast expression plasmids were transformed into S. cerevisiae S288C using the Yeast-1 transformation kit (Sigma-Aldrich, Australia), according to the provided protocol. Transformants were screened on yeast minimal growth agar supplemented with $2 \%$ agar, $2 \%$ glucose, and an amino acid mixture lacking uracil ( $\mathrm{SC}^{-}$-Ura Glu). Successful transformants were confirmed by colony polymerase chain reaction.

Starter cultures of S288C transformants were set up in $5 \mathrm{~mL}$ of $\mathrm{SC}^{-}$-Ura medium supplemented with $2 \%$ glucose. After incubation overnight at $28{ }^{\circ} \mathrm{C}$ while being shaken, yeast cells were washed with sterile deionized water. The washed yeast cells were resuspended in $5 \mathrm{~mL}$ of $\mathrm{SC}^{-}$-Ura medium supplemented with $1 \%$ Tergitol, $2 \%$ galactose, $0.25 \mathrm{mM} \alpha$ linolenic acid $\left(\mathrm{C} 18: 3 \mathrm{n}-3^{\Delta 9,12,15}\right)$, and $0.25 \mathrm{mM}$ linoleic acid $\left(\mathrm{C} 18: 2 \mathrm{n}-6^{\Delta 9,12}\right.$ ) at an $\mathrm{OD}_{600}$ of $\sim 0.3$. After overnight incubation at $28{ }^{\circ} \mathrm{C}$ while being shaken, the yeast cells were washed successively with $5 \mathrm{~mL}$ of $1 \%$ Tergitol, $0.5 \%$ Tergitol, 
A

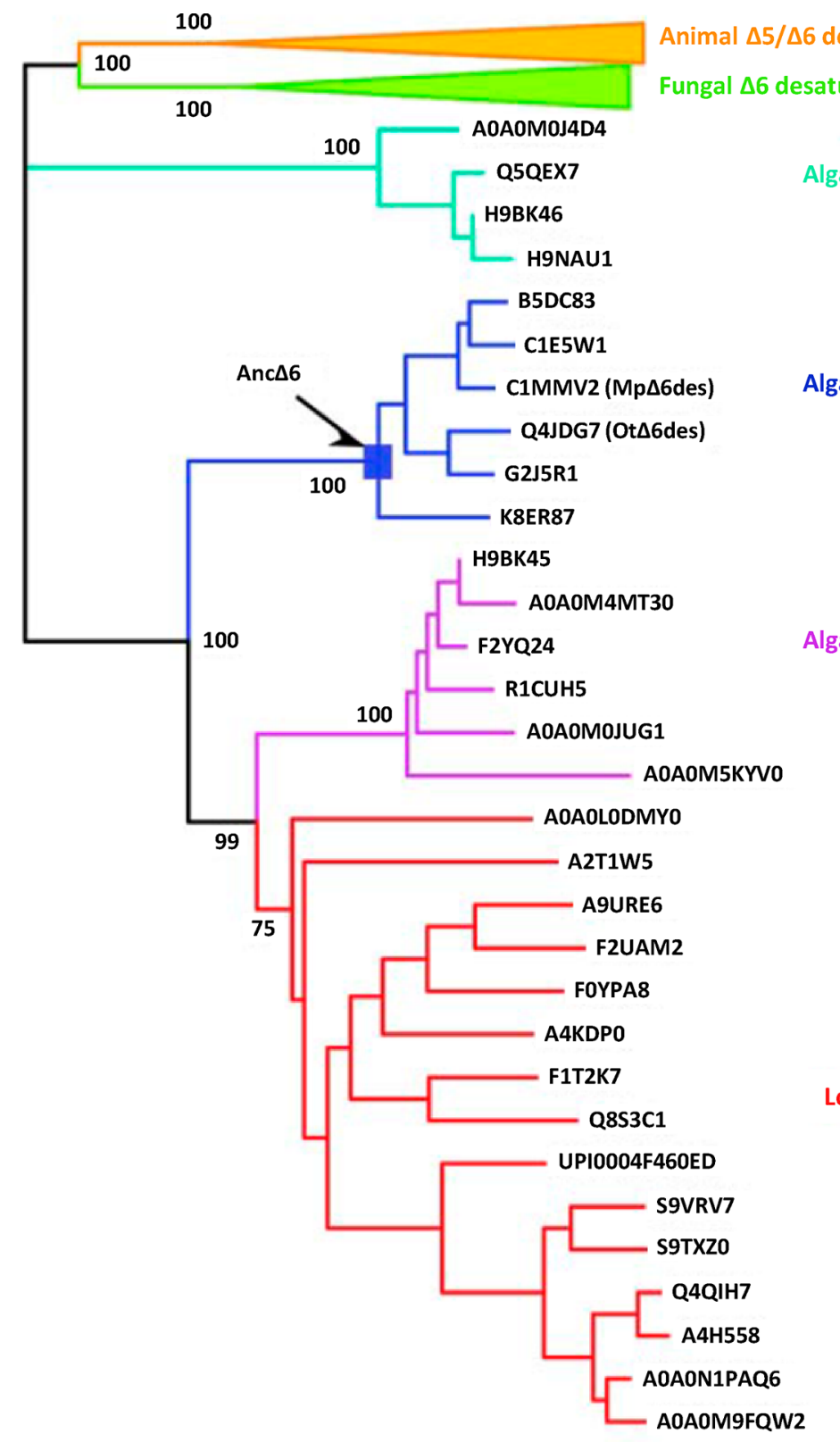

desaturases

Algal $\Delta 4$ desaturases

Algal $\Delta 6$ desaturases

Algal $\Delta 5$ desaturases

B

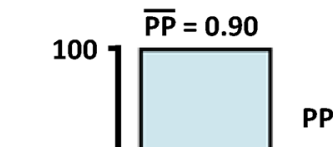

ㅁ $0.9-1.0$

ㅁ $0.8-0.9$

口 $0.7-0.8$

ㅁ $0.6-0.7$

ㅁ $0.5-0.6$

口 $0.4-0.5$

口 $0.3-0.4$

口 $0.2-0.3$

口 $0.1-0.2$

$0.0-0.1$

Lower Eukaryote $\Delta 5 / \Delta 6$ desaturases

Figure 1. Phylogenetic analysis of desaturases. (A) An unrooted phylogeny tree was constructed using MEGA version 6. The analysis demonstrated the clear phylogenetic separation of algal, fungal, and animal desaturases. Desaturase sequences included in the tree are identified in Supplementary Table 1. The bootstrap values (100 bootstrap repetitions) of major nodes are denoted. The identity of the protein at each node is presented using its UniProt ID with $\mathrm{Mp} \Delta 6 \mathrm{des}$ and Ot $\Delta 6 \mathrm{des}$ denoted for the sake of clarity. (A) The posterior probability distribution and mean posterior probability of the inferred $\Delta 6$-desaturase ancestor Anc $\Delta 6$ are shown as a cumulative probability graph for posterior probability intervals of 0.1 .

and deionized water to remove non-incorporated free fatty acids. The yeast pellets were freeze-dried for lipid analysis.

HA Tagging of Wild-Type Mp $\Delta 6$ des and Its Variants. Primers were designed to add an influenza hemagluttinin (HA) tag onto the $\mathrm{N}$-terminus of $\mathrm{Mp} \Delta 6$ des via blunt-end ligation. The $\mathrm{N}$-terminal HA-tagged variants were generated by assembling the $\mathrm{N}$-terminus of the tagged WT Mp $\Delta 6$ des with the variant genes and the KpnI/SacI-digested pYES2 vector using an in-house Gibson assembly kit. The tagged coding regions were confirmed by sequencing.

Lipid Analysis. Fatty acid methyl esters were prepared from the freeze-dried yeast pellets by transesterification with 1 $\mathrm{mL}$ of the methylation solution (10:1:1 MeOH:dichlorome- thane: $\mathrm{HCl}$ ) for $2 \mathrm{~h}$ at $80{ }^{\circ} \mathrm{C}$. After the addition of $1 \mathrm{~mL}$ of deionized water into each sample, the methylated fatty acids were extracted with $0.3 \mathrm{~mL}$ of the extraction solution (4:1 hexane:dichloromethane) and reconstituted in hexane before GC analysis. ${ }^{4}$ The desaturation efficiency was calculated on the basis of the integrated peak area as described previously ${ }^{4}$ and normalized using the wild-type desaturase result in the same assay set.

Western Blot Analysis. The S288C transformant cultures were prepared as for the feeding assay, except that Tergitol and fatty acid substrates were not added to the induced culture. The yeast cell pellets were washed with $1 \mathrm{~mL}$ of $0.1 \mathrm{M}$ Tris$\mathrm{HCl}$ ( $\mathrm{pH} 7.2$ ). The yeast lysate was prepared using $0.5 \mathrm{~mm}$ 


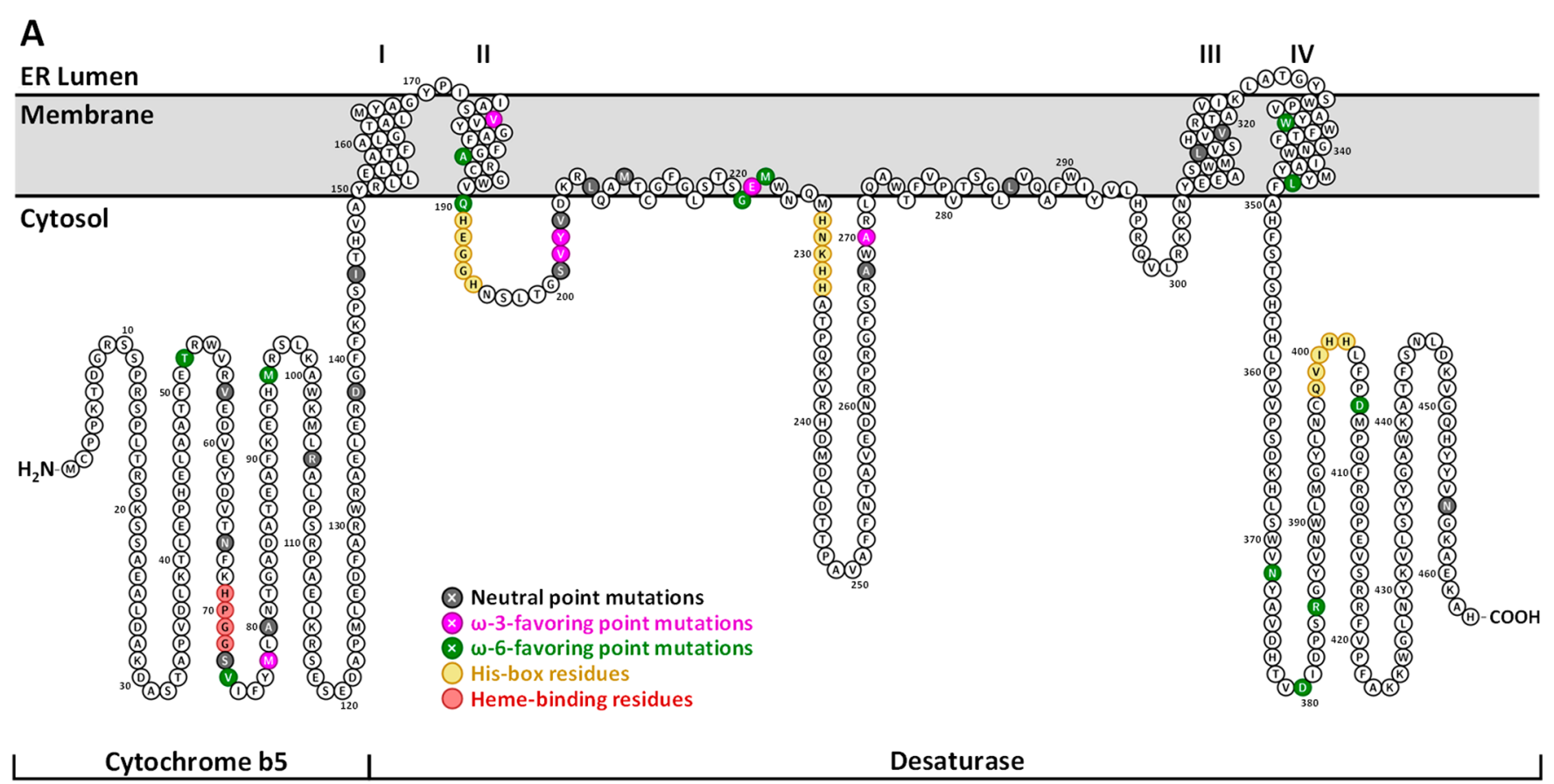

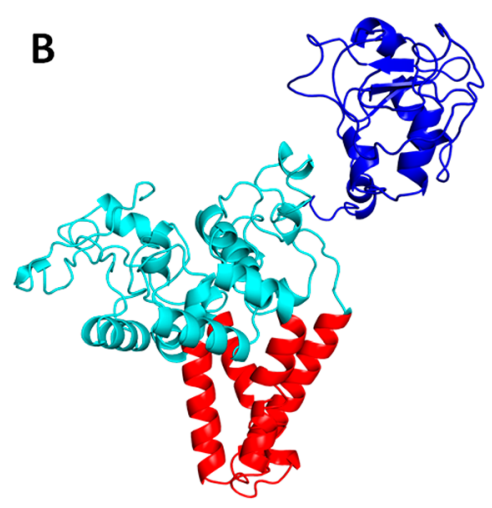

Mp $\Delta 6$ des

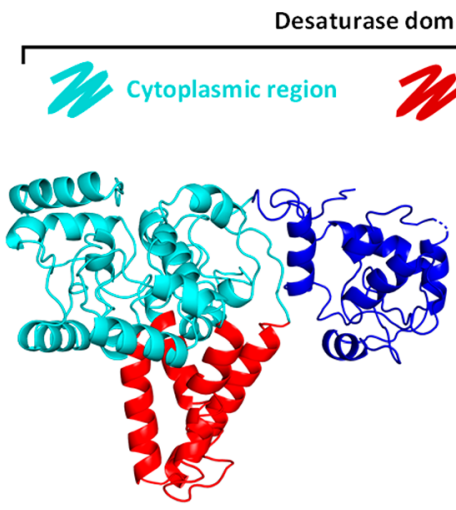

Ot $\Delta 6$ des
Transmembrane region

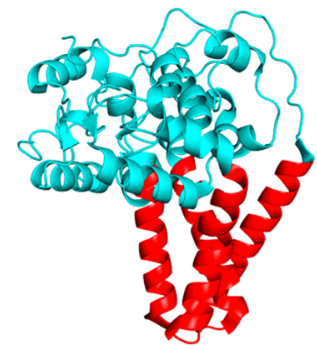

$\mathrm{Mm} \Delta 9 \mathrm{des}$ (PDB ID: 4YMK)
Cytochrome b5 domain

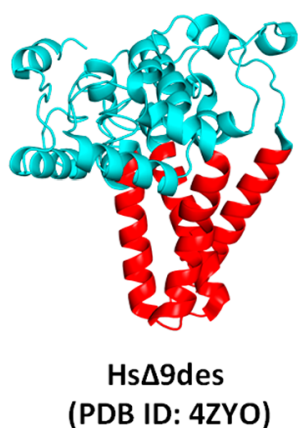

Figure 2. Predicted topology of Mp $\Delta 6$ des. (A) The corrected four-TMH topology for algal $\Delta 6$-desaturases is shown as a PROTTER topology schematic. The $\mathrm{N}$ - and C-termini as well as the His box motifs indicated are known to be cytoplasmic. (B) Phyre 2 models of Mp $\Delta 6 \mathrm{des}$ and Ot $\Delta 6 \mathrm{des}$ are shown side by side with the crystal structures of $\mathrm{Mm} \Delta$ 9des and $\mathrm{Hs} \Delta$ 9des. All four desaturases adopt a four-TMH topology. However, $\mathrm{Mm} \Delta 9 \mathrm{des}$ and $\mathrm{Hs} \Delta 9 \mathrm{des}$ do not possess a cytochrome $b_{5}$ domain; therefore, despite the correct identification of the Mp $\Delta 6 \mathrm{des}$ and Ot $\Delta 6 \mathrm{des} b_{5}$ domains by the Phyre2 algorithm, their exact position relative to the linked desaturase domain in each protein is unknown.

zirconium oxide beads and a Bullet blender (Next Advance, Cambridge, MA) in $200 \mu \mathrm{L}$ of resuspension buffer [ $50 \mathrm{mM}$ Tris-HCl (pH 7.9), $20 \mathrm{mM}$ glycerol, and $1 \mathrm{mM} \mathrm{DTT}$. The total protein concentration was determined using the Bradford Protein Assay Kit (Thermo Scientific, Waltham, MA) with albumin as a standard for each Western blot.

A sample of protein lysate equivalent to $40 \mu \mathrm{g}$ of total protein was run on a NuPAGE $10 \%$ Bis-Tris Protein Gel (NOVEX, Thermo Fisher Scientific) and transferred to a piece of a nitrocellulose membrane using a semidry transfer apparatus (Hoefer Semiphor, Pharmacia). The membrane was blocked for $1 \mathrm{~h}$ with blocking buffer [5\% skim milk suspension in $20 \mathrm{mM}$ Tris- $\mathrm{HCl}, 8 \% \mathrm{NaCl}$, and $0.1 \%$ Tween 20 $(\mathrm{pH}$ 7.6) $]$ and probed with an anti-HA antibody at a 1:5000 dilution (Sigma) in blocking buffer. The membrane was then washed four times with $20 \mathrm{~mL}$ of TBST [ $20 \mathrm{mM}$ Tris- $\mathrm{HCl}, 8 \%$ $\mathrm{NaCl}$, and $0.1 \%$ Tween $20(\mathrm{pH} \mathrm{7.6)}$ ) for $10 \mathrm{~min}$ and probed with the anti-mouse horseradish peroxidase-conjugated antibody at a 1:10000 dilution in blocking buffer. The membrane was washed four times again as before, and a 1:1 mixture of a peroxide solution and the luminol/enhancer solution from the SuperSignalchemiluminescent substrate kit (Pierce) was added. The blot was visualized with ChemiDoc (Bio-Rad).

\section{RESULTS}

Phylogenetic Analysis of Mps6des Homologues. In the absence of any molecular structure to guide mutagenesis, we first sought to obtain deeper insight into these proteins through phylogenetic analysis. Previous phylogenetic studies of membrane-bound desaturases revealed the evolutionary separation of acyl-CoA desaturases and acyl-lipid desaturases. ${ }^{4}$ For the purpose of consensus mutation design and ancestral protein reconstruction (APR), we conducted a focused phylogenetic analysis of $\mathrm{Mp} \Delta 6 \mathrm{des}$ homologues. The protein 


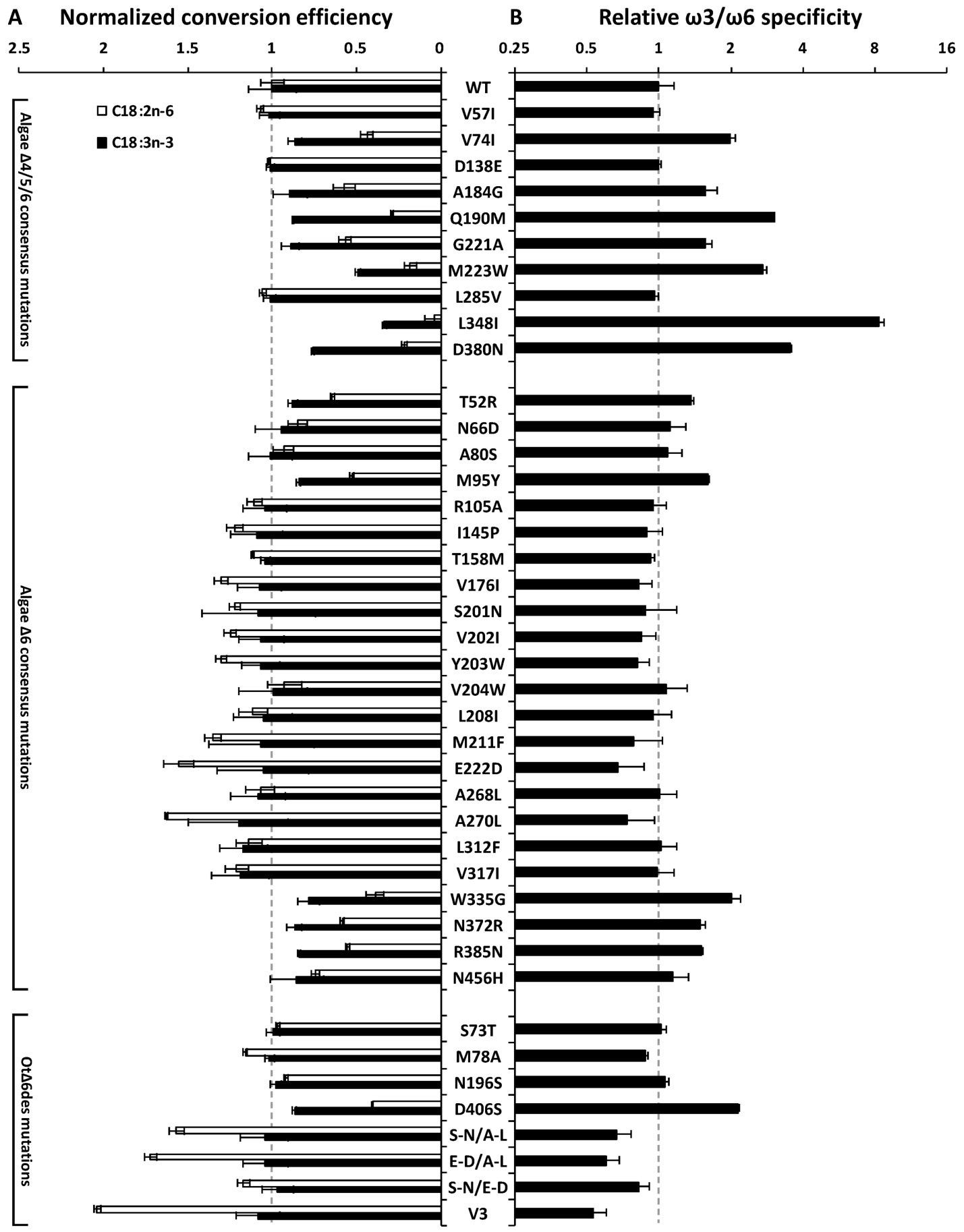

Figure 3. Activity and specificity of $\mathrm{Mp} \Delta 6$ des consensus variants. (A) Conversion efficiencies for both $\omega 3$ - and $\omega 6$-substrates (ALA, black; LA, white), defined as the percentage of $\omega 3$ - or $\omega 6$-substrate converted to products of $\Delta 6$-desaturase-dependent pathways, are shown for the Mp $\Delta 6 \mathrm{des}$ variants identified by consensus mutagenesis, normalized to wild-type conversion efficiency $(n=3 \pm 1 \mathrm{SD})$. Results are grouped by the consensus set in which they were predicted. Combinatorial mutants, grouped with Ot $\Delta 6 \mathrm{des}$, are named by the mutations included, with S-N representing the S201N mutation, E-D the E222D mutation, and A-L the A270L mutation, with the exception of V3, which includes all three mutations mentioned above. (B) Relative $\omega 3 / \omega 6$ specificities for all variants are shown, normalized to the $\omega 3: \omega 6$ ratio of the wild type $(n=3 \pm 1 \mathrm{SD})$. A ratio of $>1$ represents an increase in $\omega 3$ specificity, whereas a ratio of $<1$ represents an increase in $\omega 6$ specificity, relative to that of wild-type Mp $\Delta 6$ des.

basic local alignment search tool (BLAST) was used to search the nonredundant protein database with the $\mathrm{Mp} \Delta 6 \mathrm{des}$ protein sequence. $^{34}$ A cutoff of $26 \%$ sequence identity to $M p \Delta 6$ des was applied to obtain a data set of closely related sequences containing $\Delta 4$-, $\Delta 5$-, and $\Delta 6$-desaturases from animals, simple unicellular organisms, and marine green algae (Supplementary Table 1). No close homologues were found in modern plants.
The phylogenetic analysis of the members mentioned above using a maximum-likelihood algorithm showed the clear separation of animal desaturases from the $\Delta 5$ - and $\Delta 6$ desaturases of other organisms (Figure 1). In each group, there are separate $\Delta 5$-desaturase and $\Delta 6$-desaturase clades. Algal $\Delta 6$-desaturases form one clade, whereas the $\Delta 5$ clade contains $\Delta 5$-desaturases from algae and a number of other related 
A

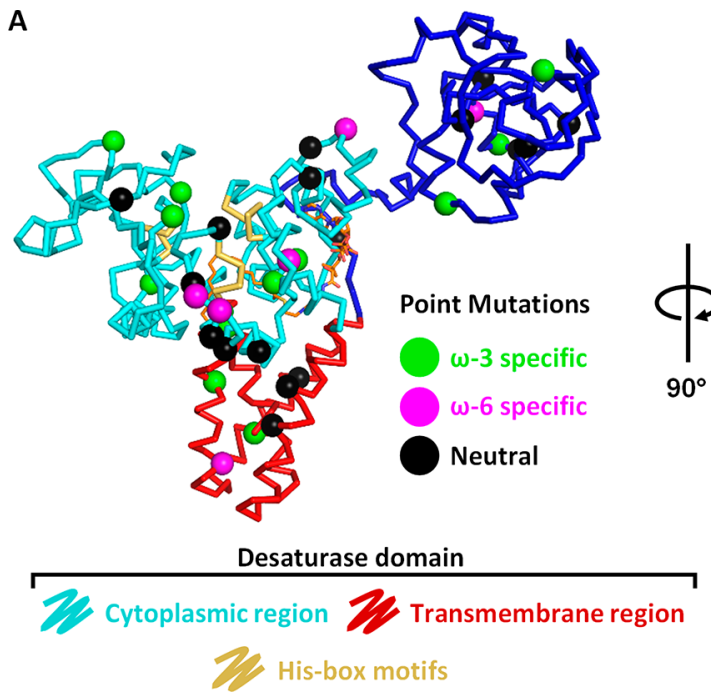

B

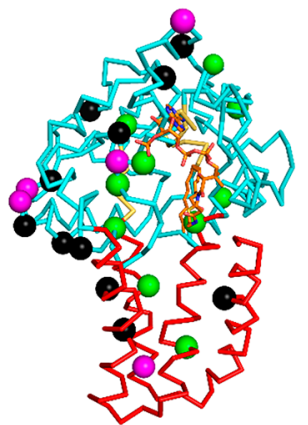

Cytochrome b5 domain

Figure 4. Position of specificity-altering $\mathrm{Mp} \Delta 6 \mathrm{des}$ mutations. (A) Ribbon representation of $\mathrm{Mp} \Delta 6 \mathrm{des}$ with mutated residues identified as colored spheres as per their effect on enzyme substrate specificity (enhancing $\omega 3$ specificity, enhancing $\omega 6$ specificity, or having no substantial effect on specificity). The conserved His box motifs are colored yellow, and a $[(Z)$-octadec-9-enyl] (2R)-2,3-bis(oxidanyl)propanoate substrate analogue, shown as orange sticks, is modeled bound to the enzyme active site based on analogue-bound structures of Mm $\Delta 9$ des and Hs $\Delta 9$ des. Several mutations cluster near either the His box motifs or the substrate binding site. Others, however, are located in distal positions such as the cytochrome $b_{5}$ domain. (B) A rotated view of the $\mathrm{Mp} \Delta 6 \mathrm{des}$ ribbon with the cytochrome $b_{5}$ domain hidden for the sake of clarity highlights the position of the substrate binding cleft.

unicellular organisms. ${ }^{46} \Delta 4$-Desaturases are present in only algae and form a distinct clade from the other algal desaturases. Considering that $\Delta 4$-desaturation is the last step of $\omega 3$ LCPUFA biosynthesis that converts DPA to DHA (Supplementary Figure 1 ), the clustering of algal $\Delta 4$-desaturases may indicate a later gene duplication event as part of cold adaptation, because the increased level of desaturation of the C22 chain could result in higher membrane fluidity. ${ }^{4-50}$ Despite the evolution of land plants from an algal common ancestor, the rare higher-plant $\Delta 6$-desaturases are thought to have originated through retrotranscription, rather than by direct inheritance. ${ }^{51}$ Hence, the absence of a modern plant acyl-CoA $\Delta 6$-desaturase homologue is not surprising.

Topology Prediction of Mps6des. Although the algal $\Delta 6$-desaturases have been functionally characterized, we understand little about how their function relates to their structure. ${ }^{4,28,30}$ Only two classes of membrane-bound desaturase topologies have been experimentally verified. Most eukaryotic desaturases adopt a four-TMH topology, including the Mus musculus and Homo sapiens $\Delta 9$ acyl-CoA desaturases $(\mathrm{Mm} \Delta$ 9des and $\mathrm{Hs} \Delta$ 9des, respectively), which are the only two membrane-bound desaturases for which high-resolution crystal structures have been determined (PDB entries 4YMK and $4 \mathrm{ZYO}$, respectively). ${ }^{42,52}$ A second topology, first validated in the Bacillus subtilis $\Delta 5$ acyl-lipid desaturase (Bs $\Delta 5 \mathrm{des}$ ), possesses six TMHs instead. ${ }^{53}$ Although these three proteins almost certainly evolved from a common ancestor and share conserved functional motifs that include their active site and the three His boxes, all three show substantial sequence variation from $\mathrm{Mp} \Delta 6 \mathrm{des}$, ranging from $16 \%$ to $20 \%$ identity (Supplementary Figure 2). To investigate the structure of $\mathrm{Mp} \Delta 6 \mathrm{des}$ and its interaction with the membrane, we performed a transmembrane helix prediction using the CCTOP topology prediction server, ${ }^{39}$ which predicts membrane protein topology via the consensus of 10 different topology prediction models enhanced with available structural information from the TOPDB protein topology database.
The CCTOP consensus predicts three putative TMHs in $\mathrm{Mp} \Delta 6$ des. However, unlike control predictions made for $\mathrm{Mm} \Delta$ 9des and Bs $\Delta 5$ des, which are accurately predicted as possessing four and six TMHs, respectively, across the majority of the modeling methods and cross-references used by CCTOP, the level of agreement between models for Mp $\Delta 6 \mathrm{des}$ is weaker, with the 10 methods predicting between three and seven TMHs (Supplementary Figure 3). In addition, the proposed three-TMH consensus cannot be accurate, as all three His boxes (positions 191-195, 228-232, and 398-402) and both termini are known to be cytoplasmic. ${ }^{10,11,46,54}$ Across all 10 models as well as in the consensus topology, however, TMHs are predicted to occur in roughly the same positions as in $\mathrm{Mm} \Delta$ 9des. It is therefore likely that the true topology of $\mathrm{Mp} \Delta 6$ des possesses four TMHs, with the predicted TMH1 helix instead forming adjacent TMH1 and TMH2 helices similarly to $\mathrm{Mm} \Delta$ 9des and $\mathrm{Hs} \Delta$ 9des (Figure $2 \mathrm{~A}$ ). We then used the Phyre $2 \mathrm{Web}$ server ${ }^{41}$ to generate homology models of $\mathrm{Mp} \Delta 6 \mathrm{des}$ and Ot $\Delta 6 \mathrm{des}$ (Figure $2 \mathrm{~B}$ ). Both resulting models utilize the existing $\Delta 9$ desaturase and cytochrome $b_{5}$ crystal structures, further suggesting homology to these four TMH desaturases, and place the four $\mathrm{Mp} \Delta 6 \mathrm{des} T \mathrm{TMH}$ at positions 150-169, 173-189, 305-323, and 329-349.

Identifying Determinants of $M p \Delta 6$ des Specificity through Consensus Mutagenesis. $\Delta 6$-Desaturases from different organisms are known to catalyze $\Delta 6$-desaturation of different acyl chains, with $\mathrm{Mp} \Delta 6 \mathrm{des}$, for example, exhibiting high specificity for $\omega 3$-substrates whereas Ot $\Delta 6$ des demonstrates comparable activity on both $\omega 3$ - and $\omega 6$-fatty acids. Rationally inferring and engineering the determinants of desaturase specificity is however a difficult task given experimental limitations when working with desaturases. A lack of available high-resolution structural information prevents the use of computational design tools to predict specificitylinked residues in $\Delta 6$-desaturases, and the low-throughput nature of existing activity assays for these enzymes precludes the use of specificity-altering methodologies that require the 
screening of a large number of mutants such as directed evolution. We thus turned to consensus mutagenesis to locate positions responsible for $\mathrm{Mp} \Delta 6 \mathrm{des}$ substrate specificity, as introducing high-probability mutations from the desaturase family in the context of the $\mathrm{Mp} \Delta 6$ des scaffold is likely to yield stable and functional enzymes, and these mutations are also more likely to encode specificity information than random mutations.

For this purpose, we used three different amino acid consensus sets to identify suitable mutation sites. The first, based on an alignment of algal $\Delta 4-, \Delta 5$-, and $\Delta 6$-desaturases (including the $\Delta 5$-desaturases from other unicellular organisms), identified 10 residues that are highly conserved throughout the alignment but differ in $\mathrm{Mp} \Delta 6 \mathrm{des}$ (Supplementary Figure 4). The second, based on an alignment of the six identified algal $\Delta 6$-desaturases (Supplementary Figure 5), identified a further 23 residues that are highly conserved but not present in $\mathrm{Mp} \Delta 6 \mathrm{des}$. Finally, to investigate the difference in specificity between $\mathrm{Mp} \Delta 6 \mathrm{des}$ and Ot $\Delta 6 \mathrm{des}$, as $\mathrm{Ot} \Delta 6 \mathrm{des}$ is the characterized algal $\Delta 6$-desaturase whose substrate specificity is most different from that of $\mathrm{Mp} \Delta 6 \mathrm{des}$, we identified four additional residues located in functionally relevant regions (near the heme binding region of the cytochrome $b_{5}$ domain or the His boxes of the desaturase domain) that vary between these proteins (Supplementary Figure 6).

Following identification of these putative specificity-linked positions, a series of $\mathrm{Mp} \Delta 6 \mathrm{des}$ single mutants were generated by reverting the $M p \Delta 6$ des amino acid at each position to the consensus amino acid. The resulting variants were expressed in yeast and compared to wild-type $\mathrm{Mp} \Delta 6 \mathrm{des}$ using a yeast competition assay to determine the effect of these mutations on enzyme activity against $\omega 3$ - and $\omega 6$-substrates [C18:3n$3^{\Delta 9,12,15} \alpha$-linolenic acid (ALA) and C18:2n- $6^{\Delta 9,12}$ linoleic acid (LA), respectively] (Figure 3A, Supplementary Figures 7 and 8 , and Supplementary Table 2). As $\Delta 6$-desaturases, including $\mathrm{Mp} \Delta 6 \mathrm{des}$, cannot be readily purified in an active form, ${ }^{53}$ whole-cell measured activity values (triplicate) were normalized against a wild-type $\mathrm{Mp} \Delta 6 \mathrm{des}$ control included in every set of assays. The level of $\Delta 6$-desaturase expression was determined using Western blots and was comparable to the level of wild-type $\mathrm{Mp} \Delta 6 \mathrm{des}$ expression for all tested variants (Supplementary Figure 7). Nonetheless, the ratio of the 133 / $\omega 6$ activities, which is unaffected by expression or activity levels, was used to determine the enzyme specificity (Figure 3B and Supplementary Table 2). Of 37 point mutations, 13 were shown to increase $\omega 3$ specificity by at least one standard deviation (SD), six were shown to increase $\omega 6$ specificity by at least one SD, and 18 were neutral (within one SD) in terms of their effect on substrate specificity.

Mapping mutation sites and effects onto our $\mathrm{Mp} \Delta 6 \mathrm{des}$ model (Figures 2A and 4A) shows one cluster of specificitymodifying mutations at positions 221-223 along with a dearth of nearby neutral mutations. This site lies in the enzyme's substrate binding cleft near the triad of His box motifs and can therefore be easily rationalized as affecting specificity (Figure 4B). This result is also consistent with previous studies of front-end desaturases, which demonstrated that mutations near His boxes could affect either substrate preference or the regioselectivity. ${ }^{56}$ The remaining mutations partition throughout the $\mathrm{Mp} \Delta 6 \mathrm{des}$ structure displaying no clear pattern, highlighting the complex nature of the interactions governing desaturase substrate specificity. However, four specificitylinked mutations are found in the $\mathrm{Mp} \Delta 6$ des cytochrome $b_{5}$ domain, such as the V74I mutation that nearly doubles the enzyme $\omega 3: \omega 6$ activity ratio relative to that of the wild type. Although the importance of the cytochrome $b_{5}$ domain has been well documented in regard to electron transport during $\Delta 6$-desaturase catalysis, knowledge of specific interactions between the cytochrome $b_{5}$ and desaturase domains remains elusive as the only structures of membrane-bound desaturases determined to date do not contain a cytochrome $b_{5}$ domain. These results are thus, to the best of our knowledge, the first evidence of a link between this domain and $\Delta 6$-desaturase substrate specificity. This is consistent with evidence that the front-end desaturases arose from an early fusion event with a cytochrome $b_{5}$ protein, followed by the co-evolution of the cytochrome $b_{5}$ domain with the desaturase, ${ }^{57}$ to form a cooperative substrate binding site.

In addition to the 37 single-point mutants characterized above, we also generated three double mutants to better understand potential interacting positions throughout the $\mathrm{Mp} \Delta 6$ des desaturase domain, each containing two of the three mutations among S201N, E222D, and A270L. These mutations were chosen as they are all found in Ot $\Delta 6 \mathrm{des}$ and individually increase $\omega 6$ activity. The resulting double mutants all demonstrate decreased $\omega 3$ specificity, with an additive effect observed when the A270L mutation is combined with either S201N or E222D. The effects of S201N and E222D on the other hand are not additive, with the S201N/E222D double mutant showing no significant changes in specificity compared to that of the S201N mutant alone. Introducing all three mutations into $\mathrm{Mp} \Delta 6 \mathrm{des}$ however nonetheless leads to a further increased $\omega 6$ specificity compared to that of any double mutant, generating the least specific $\mathrm{Mp} \Delta 6$ des variant tested and highlighting the complex interactions responsible for Mp $\Delta 6$ des specificity.

Effects of Substrate Conformational Restriction on $\Delta 6$-Desaturase Specificity. Along with identifying specificity-linked positions in $\Delta 6$-desaturases, our kinetic results (Figure 3) show a distinct correlation between activity and specificity in these enzymes. Almost universally among tested mutations, those that further increase the $\omega 3$ specificity of $\mathrm{Mp} \Delta 6$ des come at a cost to overall enzyme activity against both $\omega 3$ - and $\omega 6$-substrates, while mutations that reduce $\omega 3$ specificity lead to increased $\omega 6$ activity rather than reduced $\omega 3$ activity. This correlation highlights the difficulty the enzyme faces in discriminating between ALA and LA. Both are acyl chains of the same length, differing by only a single double bond that imparts additional rigidity and forces chain curvature into ALA compared to the more flexible LA. Strikingly, $\mathrm{Mp} \Delta 6$ des catalysis is far more efficient on the more conformationally restricted $\omega 3$-substrate, suggesting that specificity in $\mathrm{Mp} \Delta 6$ des and related desaturases is gated by substrate entropy and that conformational preorganization is crucial to efficient catalysis. Thus, further increases to $\omega 3$ specificity likely occur through further conformational restriction of the substrate via exclusion of conformational states that are accessible to $\omega 6$-substrates but are poorly populated in the more rigid $\omega 3$-substrates, in turn leading to overall decreased rates of catalysis. Likewise, a decreased $\omega 3$ specificity would occur through reduced conformational stringency, increasing $\omega 6$ activity without adversely affecting $\omega 3$ activity.

Reconstruction of an Ancestral $\Delta 6$-Desaturase. Beyond the contributions of individual positions to specificity, the results of our consensus mutagenesis studies also hint at 
A
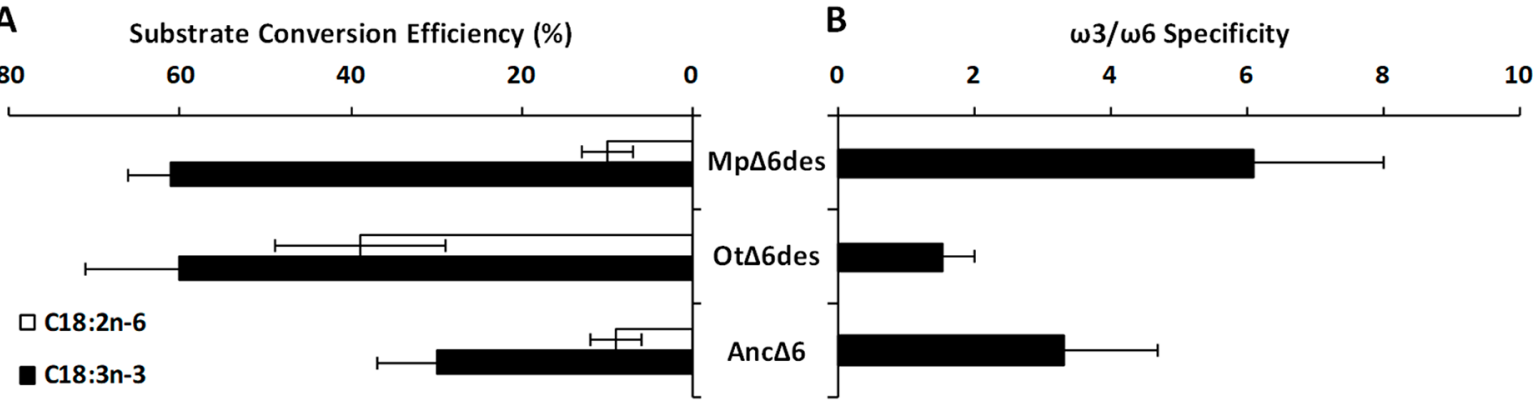

C

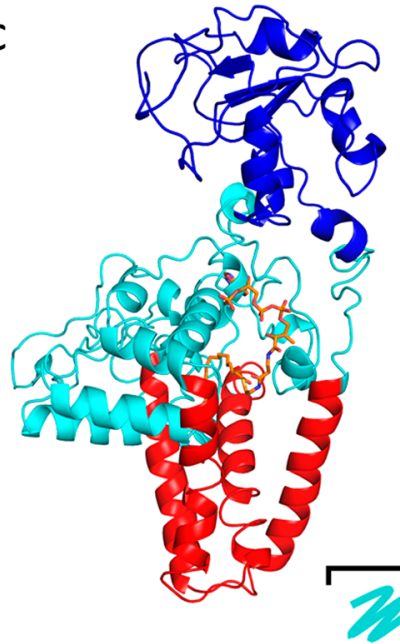

D<smiles>[2H]I</smiles>

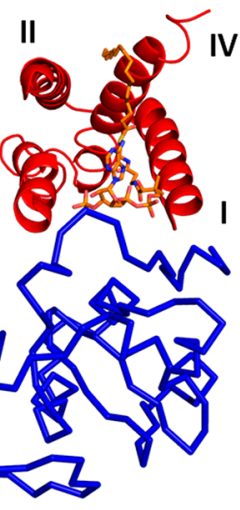

$\mathbf{F}$

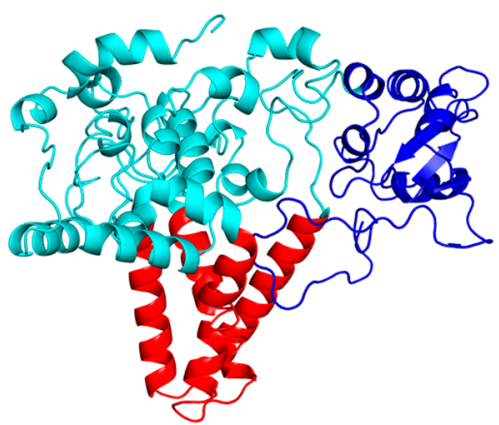

Anc $\Delta 6$

E

Cytoplasmic region

Transmembrane region

Cytochrome b5 domain

\begin{tabular}{ccccccc}
\hline \multirow{2}{*}{ Protein } & Native & \multicolumn{4}{c}{ Grand Average of Hydropathy } \\
& Substrate & TMH 1 & TMH 2 & TMH 3 & TMH 4 \\
\hline MmSCoADes & 18C:0n & 1.87 & 1.10 & 1.40 & 1.03 \\
HsSCoADes & $18 \mathrm{C}: 0 \mathrm{n}$ & 1.49 & 0.98 & 1.32 & 1.31 \\
Mp $\triangle 6$ Des & $18 \mathrm{C}: 3 \mathrm{n}-3$ & 1.23 & 0.99 & 0.86 & 0.57 \\
Ot $\triangle 6$ Des & $18 \mathrm{C}: 3 \mathrm{n}-3 /$ & 0.75 & 1.03 & 0.88 & 0.72 \\
ANC175 & $18 \mathrm{C}: 2 \mathrm{n}-6$ & $18 \mathrm{C}: 3 \mathrm{n}-3$ & 1.08 & 0.81 & 0.64 & 0.64 \\
\hline
\end{tabular}

Figure 5. Structural insights into desaturase specificity from an ancestrally reconstructed $\Delta 6$-desaturase. (A) Conversion efficiencies for both $\omega 3$ and $\omega 6$-substrates (ALA, black; LA, white) are shown for characterized $\Delta 6$-desaturases $(n=3 \pm 1 \mathrm{SD}$ ). (B) Relative $\omega 3 / \omega 6$ specificities for characterized $\Delta 6$-desaturases are shown $(n=3 \pm 1 \mathrm{SD})$, with Anc $\Delta 6$ displaying strong $\omega 3$ specificity, albeit less so than Mp $\Delta 6$ des. (C) The substrate binding pocket of $\Delta 6$-desaturases is formed by TMHs 1,3 , and 4 at the enzyme's surface, with the cytochrome $b_{5}$ domain positioned nearby due to its direct link to TMH 1. A [(Z)-octadec-9-enyl] (2R)-2,3-bis(oxidanyl)propanoate substrate analogue molecule is modeled bound to the enzyme active site based on analogue-bound structures of $\mathrm{Mm} \Delta$ 9des and Hs $\Delta$ 9des. (D) TMH numbering highlighting the orientation of helices around the substrate binding pocket. The $b_{5}$ domain is shown as a ribbon to indicate its relative position. (E) The grand average of hydropathy models based on the primary sequence of the four TMHs shows a decreased hydropathy for TMHs 1, 3, and 4 in $\Delta 6$-desaturases relative to control $\Delta$ 9-desaturases. (F) The Phyre2 model of Anc $\Delta 6$ demonstrates a potential interaction between the $\mathrm{N}$-terminal loop of the cytochrome $b_{5}$ domain in $\Delta 6$-desaturases and their substrate binding cleft.

how substrate specificity evolved among members of the desaturase family. Although stemming from the larger set of sequences, mutations belonging to the consensus set of $\Delta 4$ / $\Delta 5 / \Delta 6$-desaturases are neutral or enhance $\omega 3$ specificity (three neutral and seven $\omega 3$-specific mutations), suggesting an evolutionary bias toward $\omega 3$-substrates among front-end desaturases. Mutations from the set of algal $\Delta 6$-desaturases, however, do not bias toward either LA or ALA specificity (13 neutral, five $\omega 3$-specific, and five $\omega 6$-specific mutations). In addition, the four characterized members of the $\Delta 6$-desaturase clade display vastly different specificities, from ALA and LA conversion efficiencies of $63 \%$ and $5 \%$ for $\mathrm{Mp} \Delta 6 \mathrm{des}$ to $71 \%$ and $73 \%$ for Ot $\Delta 6$ des, respectively (Supplementary Table 3 ). To address the question of whether the $\omega 3$ specificity of 
$\mathrm{Mp} \Delta 6 \mathrm{des}$ or the substrate promiscuity of Ot $\Delta 6 \mathrm{des}$ is a recently diverged phenotype, the substrate specificity of the putative common ancestor of the algal $\Delta 6$-desaturase family was tested. This ancestor, Anc $\Delta 6$, was inferred from the algal $\Delta 6$ clade juncture using a phylogenetic tree (Figure 1 ). The mean posterior probability of the resulting Anc $\Delta 6$ sequence of 0.90 is comparable to that of other successfully reconstructed ancestral proteins. ${ }^{58}$ Notably, Anc $\Delta 6$ exhibits greater fulllength expression than the wild-type proteins, which is consistent with previous examples of proteins generated through ancestral sequence reconstruction (Supplementary Figure 7). ${ }^{58}$ Anc $\Delta 6$ displays activity comparable to, if slightly lower than, that of the wild-type proteins and is specific for $\omega 3$ fatty acids, albeit less specific than $\mathrm{Mp} \Delta 6$ des (Figure 5A,B). This suggests that the substrate promiscuity toward $\omega 3 / \omega 6$ substrates observed in Ot $\Delta 6 \mathrm{des}$ could be a more recent adaptation in response to environmental changes. Notably, the sequence differences between Anc $\Delta 6$ and $M p \Delta 6$ des tend to cluster in the cytochrome $b_{5}$ domain and the cytosolic loop of His Box I, implying a role for these regions in improved $\omega 3$ specificity. In contrast, the sequence differences between Anc $\Delta 6$ and Ot $\Delta 6$ des tend to cluster in the cytochrome $b_{5}$ domain and the region between His Box II and putative TMH3.

\section{DISCUSSION}

Our results demonstrate that front-end desaturase substrate specificity is the result of subtle and complex interactions between residues throughout the enzyme. Notably, we show that the cytochrome $b_{5}$ domain, previously thought to be involved primarily in electron transfer during catalysis, ${ }^{59}$ is also implicated in front-end desaturase substrate recognition. Considering the subtle structural difference between $\omega 3$ - and $\omega 6$-substrates, these results suggest that the cytochrome $b_{5}$ domain plays an additional role in refining the geometry of the substrate binding cavity and imply that it is physically part of the binding site or modifies the shape and/or nature of the binding site via outer-shell interactions. ${ }^{60}$ On the basis of homology to known desaturase structures, as well as the cytosolic position of the desaturase domain $\mathrm{N}$-terminus, we can putatively position the $b_{5}$ domain of front-end desaturases in the cytosol near the cleft formed by TMHs 1, 3, and 4 (Figure 5C,D). As this cleft contains the substrate binding pocket, the $b_{5}$ domain would thus be poised to interact with both the substrate and the desaturase domain. Further examination of the TMH sequences in front-end desaturases also reveals that these three helices are less hydrophobic than those found in characterized desaturases lacking a fused $b_{5}$ domain (Figure 5E), while TMH 2, which does not contribute to forming this cleft, is similarly hydrophobic across both sets of desaturases. This increased hydrophilicity in front-end desaturases suggests a weaker membrane interaction and also explains the difficulty in accurately predicting TMH topology in $\mathrm{Mp} \Delta 6$ des. In addition, the $\mathrm{N}$-terminal portion of the cytochrome $b_{5}$ domain in front-end desaturases shares little homology with cytochrome $b_{5}$ proteins, instead consisting of a proline and charged amino acid-rich motif resembling intrinsically disordered regions in other proteins. The conformational flexibility of such a region coupled with weaker membrane interactions in the desaturase domain could allow for interactions between the $b_{5}$ domain and the $\Delta 6$-desaturase substrate binding pocket. Such a contact was observed in the Anc 66 Phyre2 model, demonstrating potential complemen- tarity of the $b_{5} \mathrm{~N}$-terminal tail and the TMH 1, 3, 4 cleft and a potential mechanism for substrate gating by the $b_{5}$ domain (Figure 5F).

In addition to an examination of their function, our reconstruction of an ancestral $\Delta 6$-desaturase also helps us to understand how these enzymes evolved. Mp $\Delta 6$ des was originally isolated from $M$. pusilla CCMP1545, which is identified as being from the Atlantic Ocean (English Channel), ${ }^{61}$ and is one of the most ubiquitous strains of marine algae. ${ }^{62}$ However, M. pusilla CCMP1545 is absent in the oligotrophic, high salt and high light, Mediterranean Sea, where O. tauri OTTH0595 is instead abundant. ${ }^{62,63}$ Rather than geographical barriers driving divergence between species, differences among salt concentrations, light intensities, and nutrient availabilities in some habitats might be an alternative selection pressure. ${ }^{64,65}$ Increased levels of unsaturated fatty acids can maintain an adequate level of membrane fluidity and protect the photosynthesis machinery, thereby increasing the salt tolerance of photosynthetic organisms. ${ }^{47,66-68}$ Furthermore, excess photoassimilates are stored in the form of polyunsaturated fatty acids as a means of preventing oxidative stress under high-light treatment. ${ }^{6-71}$ Ot $\Delta 6$ des activity produces comparatively elevated levels of $\omega 6$-fatty acids, which can then be incorporated into membranes and contribute to an increased degree of membrane ordering. Thus, it is expected that the promiscuity of this enzyme will result in a reduction in membrane fluidity given a similar level of LCPUFA incorporation. However, the higher temperatures in the Mediterranean Sea can mitigate these effects, promoting a higher rate of overall desaturation over specificity to increase fitness in a high-light environment. The colder environment of the Atlantic, on the other hand, requires organisms to develop higher membrane fluidity, hence promoting the evolution of more stringent $\Delta 6$-desaturases that display reduced rates of $\omega 6$-fatty acid production. Thus, we propose that the high salt concentration and high light levels of the Mediterranean Sea might have driven the divergence of the $O$. tauri $\Delta 6$-desaturase for high salt and light tolerance.

\section{CONCLUSION}

Although most enzyme engineering and evolution studies use enzymes that can be readily purified or easily assayed or for which high-resolution structural information is available, desaturases present a more difficult system to study due to the lack of all three factors. To circumvent these complicating factors, we used a combination of consensus mutagenesis, modeling, and ancestral sequence reconstruction to probe desaturase activity and specificity. Of 37 consensus mutations found throughout the algal desaturase clades, all expressed and were active, and several encoded altered specificities that provided new insight into the substrate specificity of these proteins. Several of the variants that we have produced could have value for biotechnology or as the basis for future biotechbased research, including variants with $\leq 8$-fold increased $\omega$ - 3 substrate specificity (albeit with loss of activity) and increased levels of expression in yeast $(\operatorname{Anc} \Delta 6)$ that could prove to be useful, depending on the rate-limiting factors in recombinant systems. Overall, our study has revealed new and useful insight into the structure-function relationship and evolution of these useful enzymes. This highlights the power of bioinformaticsinspired engineering approaches for generating small and manageable variant libraries for enzymes that are otherwise 
difficult to engineer, while also paving the way for the future engineering of industrially relevant LCPUFA desaturases.

\section{ASSOCIATED CONTENT}

\section{SI Supporting Information}

The Supporting Information is available free of charge at https://pubs.acs.org/doi/10.1021/acs.biochem.0c00110.

Sequence alignments and sequence identities, Genbank IDs for the phylogeny tree, CCTOP topology predictions, Western blots of expressed desaturase variants, and tabulated numerical data and representative GC traces for activity and specificity measurements (PDF)

\section{Accession Codes}

Mp $\Delta 6$ des, C1MMV2; Ot $\Delta 6$ des, Q4JDG7; Mm $\Delta 9$ des, Q4DSA0; Hs $\Delta 9$ des, O00767; Bs $\Delta 5$ des, O34653; Ol $\Delta 6$ des, G2J5R1; Ms $\Delta 6$ des, B5DC83.

\section{AUTHOR INFORMATION}

\section{Corresponding Author}

Colin J. Jackson - Research School of Chemistry, The Australian National University, Canberra, ACT 2601, Australia; ARC Centre of Excellence in Synthetic Biology, Macquarie University, Sydney NSW 2109, Australia; ๑) orcid.org/0000-0001-6150-3822; Phone: +61-2-6125 8325; Email: colin.jackson@anu.edu.au; Fax: +61-2-6125 0750

\section{Authors}

Dongdi Li - Research School of Chemistry, The Australian National University, Canberra, ACT 2601, Australia

Adam M. Damry - Research School of Chemistry, The Australian National University, Canberra, ACT 2601, Australia; ○ orcid.org/0000-0003-3596-3133

James R. Petrie - CSIRO Agriculture Flagship, Black Mountain Laboratories, Canberra, ACT 2601, Australia

Thomas Vanhercke - CSIRO Agriculture Flagship, Black Mountain Laboratories, Canberra, ACT 2601, Australia

Surinder P. Singh - CSIRO Agriculture Flagship, Black

Mountain Laboratories, Canberra, ACT 2601, Australia

Complete contact information is available at:

https://pubs.acs.org/10.1021/acs.biochem.0c00110

\section{Author Contributions}

${ }^{\infty}$ D.L. and A.M.D. contributed equally to this work. D.L., C.J.J., T.V., J.R.P., and S.P.S. designed the experiments. D.L. performed all of the experiments. A.M.D., D.L., and C.J.J. analyzed data with input from other authors. A.M.D., D.L., and C.J.J. wrote the manuscript with input from all authors.

\section{Funding}

This work was funded by Commonwealth Science and Industrial Research Organisation Agriculture Flagship. D.L. was funded by an ANU Ph.D. Scholarship.

\section{Notes}

The authors declare no competing financial interest.

\section{ACKNOWLEDGMENTS}

The authors thank Mr. Adam White, Dr. Pushkar Shrestha, and Dr. Xue-Rong Zhou for their excellent technical assistance and Professor Susan Howitt for providing the Western blot apparatus and nitrocellulose membranes.

\section{REFERENCES}

(1) Lee, J. H., O’Keefe, J. H., Lavie, C. J., and Harris, W. S. (2009) Omega-3 fatty acids: cardiovascular benefits, sources and sustainability. Nat. Rev. Cardiol. 6, 753-758.

(2) Chalon, S. (2006) Omega-3 fatty acids and monoamine neurotransmission. Prostaglandins, Leukotrienes Essent. Fatty Acids 75, 259-269.

(3) Murphy, M. G. (1990) Dietary fatty acids and membrane protein function. J. Nutr. Biochem. 1, 68-79.

(4) Petrie, J. R., Shrestha, P., Mansour, M. P., Nichols, P. D., Liu, Q. and Singh, S. P. (2010) Metabolic engineering of omega-3 long-chain polyunsaturated fatty acids in plants using an acyl-CoA Delta6desaturase with omega3-preference from the marine microalga Micromonas pusilla. Metab. Eng. 12, 233-240.

(5) Petrie, J. R., Shrestha, P., Zhou, X. R., Mansour, M. P., Liu, Q., Belide, S., Nichols, P. D., and Singh, S. P. (2012) Metabolic engineering plant seeds with fish oil-like levels of DHA. PLoS One 7, No. e49165.

(6) Sayanova, O. V., Beaudoin, F., Michaelson, L. V., Shewry, P. R., and Napier, J. A. (2003) Identification of Primula fatty acid Delta(6)desaturases with n-3 substrate preferences. FEBS Lett. 542, 100-104.

(7) Tang, X., Chen, H., Mei, T., Ge, C., Gu, Z., Zhang, H., Chen, Y. Q., and Chen, W. (2018) Characterization of an Omega-3 Desaturase From Phytophthora parasitica and Application for Eicosapentaenoic Acid Production in Mortierella alpina. Front. Microbiol. 9, No. e1878.

(8) López Alonso, D., Garcia-Maroto, F., Rodriguez-Ruiz, J., Garrido, J. A., and Vilches, M. A. (2003) Evolution of the membrane-bound fatty acid desaturases. Biochem. Syst. Ecol. 31, $1111-1124$

(9) Los, D. A., and Murata, N. (1998) Structure and expression of fatty acid desaturases. Biochim. Biophys. Acta, Lipids Lipid Metab. 1394, $3-15$.

(10) Vanhercke, T., Shrestha, P., Green, A. G., and Singh, S. P. (2011) Mechanistic and Structural Insights into the Regioselectivity of an Acyl-CoA Fatty Acid Desaturase via Directed Molecular Evolution. J. Biol. Chem. 286, 12860-12869.

(11) Na-Ranong, S., Laoteng, K., Kittakoop, P., Tanticharoen, M., and Cheevadhanarak, S. (2006) Targeted mutagenesis of a fatty acid Delta6-desaturase from Mucor rouxii: role of amino acid residues adjacent to histidine-rich motif II. Biochem. Biophys. Res. Commun. 339, 1029-1034.

(12) Sayanova, O., Beaudoin, F., Libisch, B., Castel, A., Shewry, P. R., and Napier, J. A. (2001) Mutagenesis and heterologous expression in yeast of a plant Delta6-fatty acid desaturase. J. Exp. Bot. 52, 15811585.

(13) Shanklin, J., Whittle, E., and Fox, B. G. (1994) Eight histidine residues are catalytically essential in a membrane-associated iron enzyme, stearoyl-CoA desaturase, and are conserved in alkane hydroxylase and xylene monooxygenase. Biochemistry 33, 1278712794.

(14) Avelange-Macherel, M. H., Macherel, D., Wada, H., and Murata, N. (1995) Site-directed mutagenesis of histidine residues in the delta 12 acyl-lipid desaturase of Synechocystis. FEBS Lett. 361, $111-114$

(15) Lindqvist, Y., Huang, W., Schneider, G., and Shanklin, J. (1996) Crystal structure of delta9 stearoyl-acyl carrier protein desaturase from castor seed and its relationship to other di-iron proteins. EMBO J. 15, 4081-4092.

(16) Dailey, H. A., and Strittmatter, P. (1979) Modification and identification of cytochrome b5 carboxyl groups involved in proteinprotein interaction with cytochrome b5 reductase. J. Biol. Chem. 254, $5388-5396$.

(17) Hackett, C. S., and Strittmatter, P. (1984) Covalent crosslinking of the active sites of vesicle-bound cytochrome b5 and NADH-cytochrome b5 reductase. J. Biol. Chem. 259, 3275-3282.

(18) Strittmatter, P., Spatz, L., Corcoran, D., Rogers, M. J., Setlow, B., and Redline, R. (1974) Purification and Properties of Rat-Liver Microsomal Stearyl Coenzyme-a Desaturase. Proc. Natl. Acad. Sci. U. S. A. $71,4565-4569$. 
(19) Sayanova, O., Shewry, P. R., and Napier, J. A. (1999) Histidine41 of the Cytochrome b5 Domain of the Borage $\Delta 6$ Fatty Acid Desaturase Is Essential for Enzyme Activity. Plant Physiol. 121, 641646.

(20) Zhou, X. R., Robert, S. S., Singh, S. P., and Green, A. G. (2007) Heterologous production of GLA and SDA by expression of an Echium plantagineum $\Delta 6$-desaturase gene. Plant Sci. 172, 421-422.

(21) Napier, J. A., and Sayanova, O. (2005) The production of verylong-chain PUFA biosynthesis in transgenic plants: towards a sustainable source of fish oils. Proc. Nutr. Soc. 64, 387-393.

(22) Petrie, J. R., Shrestha, P., Belide, S., Kennedy, Y., Lester, G., Liu, Q., Divi, U. K., Mulder, R. J., Mansour, M. P., Nichols, P. D., and Singh, S. P. (2014) Metabolic engineering Camelina sativa with fish oil-like levels of DHA. PLoS One 9, No. e85061.

(23) Domergue, F., Abbadi, A., Zahringer, U., Moreau, H., and Heinz, E. (2005) In vivo characterization of the first acyl-CoA Delta6desaturase from a member of the plant kingdom, the microalga Ostreococcus tauri. Biochem. J. 389, 483-490.

(24) Diao, J., Song, X., Guo, T., Wang, F., Chen, L., and Zhang, W. (2019) Cellular engineering strategies toward sustainable omega-3 long chain polyunsaturated fatty acids production: State of the art and perspectives. Biotechnol. Adv., No. 107497.

(25) Song, L. Y., Zhang, Y., Li, S. F., Hu, J., Yin, W. B., Chen, Y. H., Hao, S. T., Wang, B. L., Wang, R. R., and Hu, Z. M. (2014) Identification of the substrate recognition region in the Delta-fatty acid and Delta -sphingolipid desaturase by fusion mutagenesis. Planta 239, 753.

(26) Meesapyodsuk, D., and Qiu, X. (2014) Structure Determinants for the Substrate Specificity of Acyl-CoA Delta9 Desaturases from a Marine Copepod. ACS Chem. Biol. 9, 922.

(27) Petrie, J. R., and Singh, S. P. (2011) Expanding the docosahexaenoic acid food web for sustainable production: engineering lower plant pathways into higher plants. AoB Plants 2011, No. plr011.

(28) Hoffmann, M., Wagner, M., Abbadi, A., Fulda, M., and Feussner, I. (2008) Metabolic engineering of omega3-very long chain polyunsaturated fatty acid production by an exclusively acyl-CoAdependent pathway. J. Biol. Chem. 283, 22352-22362.

(29) Hong, H., Datla, N., Reed, D. W., Covello, P. S., MacKenzie, S. L., and Qiu, X. (2002) High-level production of gamma-linolenic acid in Brassica juncea using a Delta 6 desaturase from Pythium irregulare. Plant Physiol. 129, 354-362.

(30) Petrie, J. R., Liu, Q., Mackenzie, A. M., Shrestha, P., Mansour, M. P., Robert, S. S., Frampton, D. F., Blackburn, S. I., Nichols, P. D., and Singh, S. P. (2010) Isolation and characterisation of a highefficiency desaturase and elongases from microalgae for transgenic LC-PUFA production. Mar. Biotechnol. 12, 430-438.

(31) Hitz, W. D., Carlson, T. J., Booth, J. R., Jr., Kinney, A. J., Stecca, K. L., and Yadav, N. S. (1994) Cloning of a higher-plant plastid omega-6 fatty acid desaturase cDNA and its expression in a cyanobacterium. Plant Physiol. 105, 635-641.

(32) Winston, F., Dollard, C., and Ricupero-Hovasse, S. L. (1995) Construction of a set of convenient Saccharomyces cerevisiae strains that are isogenic to S288C. Yeast 11, 53-55.

(33) Pruitt, K. D., Tatusova, T., and Maglott, D. R. (2007) NCBI reference sequences (RefSeq): a curated non-redundant sequence database of genomes, transcripts and proteins. Nucleic Acids Res. 35, D61-65.

(34) Zhang, J., and Madden, T. L. (1997) PowerBLAST: a new network BLAST application for interactive or automated sequence analysis and annotation. Genome Res. 7, 649-656.

(35) Huang, Y., Niu, B., Gao, Y., Fu, L., and Li, W. (2010) CD-HIT Suite: a web server for clustering and comparing biological sequences. Bioinformatics 26, 680-682.

(36) Edgar, R. C. (2004) MUSCLE: multiple sequence alignment with high accuracy and high throughput. Nucleic Acids Res. 32, 17921797.
(37) Tamura, K., Stecher, G., Peterson, D., Filipski, A., and Kumar, S. (2013) MEGA6: Molecular Evolutionary Genetics Analysis version 6.0. Mol. Biol. Evol. 30, 2725-2729.

(38) Yang, Z. (2007) PAML 4: phylogenetic analysis by maximum likelihood. Mol. Biol. Evol. 24, 1586-1591.

(39) Dobson, L., Remenyi, I., and Tusnady, G. E. (2015) CCTOP: A Consensus Constrained TOPology prediction web server. Nucleic Acids Res. 43, W408-W412.

(40) Omasits, U., Ahrens, C. H., Muller, S., and Wollscheid, B. (2014) Protter: interactive protein feature visualization and integration with experimental proteomic data. Bioinformatics 30, 884-886.

(41) Kelley, L. A., Mezulis, S., Yates, C. M., Wass, M. N., and Sternberg, M. J. E. (2015) The Phyre2 web portal for protein modeling, prediction and analysis. Nat. Protoc. 10, 845-858.

(42) Bai, Y., McCoy, J. G., Levin, E. J., Sobrado, P., Rajashankar, K. R., Fox, B. G., and Zhou, M. (2015) X-ray structure of a mammalian stearoyl-CoA desaturase. Nature 524, 252-256.

(43) Wang, H., Klein, M. G., Zou, H., Lane, W., Snell, G., Levin, I., Li, K., and Sang, B. C. (2015) Crystal structure of human stearoylcoenzyme A desaturase in complex with substrate. Nat. Struct. Mol. Biol. 22, 581-585.

(44) Zheng, L., Baumann, U., and Reymond, J. L. (2004) An efficient one-step site-directed and site-saturation mutagenesis protocol. Nucleic Acids Res. 32, No. e115.

(45) Gibson, D. G., Young, L., Chuang, R. Y., Venter, J. C., Hutchison, C. A., 3rd, and Smith, H. O. (2009) Enzymatic assembly of DNA molecules up to several hundred kilobases. Nat. Methods 6, $343-345$.

(46) Tripodi, K. E., Buttigliero, L. V., Altabe, S. G., and Uttaro, A. D. (2006) Functional characterization of front-end desaturases from trypanosomatids depicts the first polyunsaturated fatty acid biosynthetic pathway from a parasitic protozoan. FEBS J. 273, 271280.

(47) Sakamoto, T., and Murata, N. (2002) Regulation of the desaturation of fatty acids and its role in tolerance to cold and salt stress. Curr. Opin. Microbiol. 5, 208-210.

(48) Svensk, E., Stahlman, M., Andersson, C. H., Johansson, M., Boren, J., and Pilon, M. (2013) PAQR-2 regulates fatty acid desaturation during cold adaptation in C. elegans. PLoS Genet. 9, No. e1003801.

(49) Chinnusamy, V., Zhu, J., and Zhu, J. K. (2007) Cold stress regulation of gene expression in plants. Trends Plant Sci. 12, 444-451.

(50) Michaud, M. R., and Denlinger, D. L. (2006) Oleic acid is elevated in cell membranes during rapid cold-hardening and pupal diapause in the flesh fly, Sarcophaga crassipalpis. J. Insect Physiol. 52, $1073-1082$

(51) Garcia-Maroto, F., Garrido-Cardenas, J. A., Vilches-Ferron, M. A., Manas-Fernandez, A., and López Alonso, D. (2006) Evolution of 'front-end' desaturases in Echium (Boraginaceae). Biochem. Syst. Ecol. $34,327-337$.

(52) Ai, H. W., Shaner, N. C., Cheng, Z., Tsien, R. Y., and Campbell, R. E. (2007) Exploration of new chromophore structures leads to the identification of improved blue fluorescent proteins. Biochemistry 46, 5904-5910.

(53) Diaz, A. R., Mansilla, M. C., Vila, A. J., and de Mendoza, D. (2002) Membrane topology of the acyl-lipid desaturase from Bacillus subtilis. J. Biol. Chem. 277, 48099-48106.

(54) Li, S. F., Song, L. Y., Zhang, G. J., Yin, W. B., Chen, Y. H., Wang, R. R., and Hu, Z. M. (2011) Newly identified essential amino acid residues affecting Delta8-sphingolipid desaturase activity revealed by site-directed mutagenesis. Biochem. Biophys. Res. Commun. 416, $165-171$.

(55) Wilding, M., Nachtschatt, M., Speight, R., and Scott, C. (2017) An improved and general streamlined phylogenetic protocol applied to the fatty acid desaturase family. Mol. Phylogenet. Evol. 115, 50-57.

(56) Shi, H., Chen, H., Gu, Z., Song, Y., Zhang, H., Chen, W., and Chen, Y. Q. (2015) Molecular mechanism of substrate specificity for 
delta 6 desaturase from Mortierella alpina and Micromonas pusilla. J. Lipid Res. 56, 2309-2321.

(57) Gostincar, C., Turk, M., and Gunde-Cimerman, N. (2010) The Evolution of Fatty Acid Desaturases and Cytochrome b5 in Eukaryotes. J. Membr. Biol. 233, 63-72.

(58) Whitfield, J. H., Zhang, W. H., Herde, M. K., Clifton, B. E., Radziejewski, J., Janovjak, H., Henneberger, C., and Jackson, C. J. (2015) Construction of a robust and sensitive arginine biosensor through ancestral protein reconstruction. Protein Sci. 24, 1412-1422. (59) Napier, J. A., Michaelson, L. V., and Sayanova, O. (2003) The role of cytochrome b5 fusion desaturases in the synthesis of polyunsaturated fatty acids. Prostaglandins, Leukotrienes Essent. Fatty Acids 68, 135-143.

(60) Hong, N.-S., Petrovic, D., Lee, R., Gryn’ova, G., Purg, M., Saunders, J., Bauer, P., Carr, P. D., Lin, C.-Y., Mabbitt, P. D., Zhang, W., Altamore, T., Easton, C., Coote, M. L., Kamerlin, S. C., and Jackson, C. J. (2018) The evolution of multiple active site configurations in a designed enzyme. Nat. Commun. 9, No. 3900.

(61) Vaulot, D., Le Gall, F., Marie, D., Guillou, L., and Partensky, F. (2004) The Roscoff Culture Collection (RCC): a collection dedicated to marine picoplankton. Nova Hedwigia 79, 49-70.

(62) Vaulot, D., Eikrem, W., Viprey, M., and Moreau, H. (2008) The diversity of small eukaryotic phytoplankton ( $<$ or $=3$ microm $)$ in marine ecosystems. FEMS microbiology reviews 32, 795-820.

(63) Marie, D., Zhu, F., Balague, V., Ras, J., and Vaulot, D. (2006) Eukaryotic picoplankton communities of the Mediterranean Sea in summer assessed by molecular approaches (DGGE, TTGE, QPCR). FEMS Microbiol. Ecol. 55, 403-415.

(64) Rodriguez, F., Derelle, E., Guillou, L., Le Gall, F., Vaulot, D., and Moreau, H. (2005) Ecotype diversity in the marine picoeukaryote Ostreococcus (Chlorophyta, Prasinophyceae). Environ. Microbiol. 7, 853-859.

(65) Psarra, S., Tselepides, A., and Ignatiades, L. (2000) Primary productivity in the oligotrophic Cretan Sea (NE Mediterranean): seasonal and interannual variability. Prog. Oceanogr. 46, 187-204.

(66) Allakhverdiev, S. I., Kinoshita, M., Inaba, M., Suzuki, I., and Murata, N. (2001) Unsaturated fatty acids in membrane lipids protect the photosynthetic machinery against salt-induced damage in Synechococcus. Plant Physiol. 125, 1842-1853.

(67) Zhang, J. T., Liu, H., Sun, J., Li, B., Zhu, Q., Chen, S. L., and Zhang, H. X. (2012) Arabidopsis Fatty Acid Desaturase FAD2 Is Required for Salt Tolerance during Seed Germination and Early Seedling Growth. PLoS One 7, e30355.

(68) Klyachko-Gurvich, G. L., Tsoglin, L. N., Doucha, J., Kopetskii, J., Shebalina, I. B., and Semenenko, V. E. (1999) Desaturation of fatty acids as an adaptive response to shifts in light intensity. Physiol. Plant. $107,240-249$.

(69) Solovchenko, A. E., Khozin-Goldberg, I., Didi-Cohen, S., Cohen, Z., and Merzlyak, M. N. (2008) Effects of light intensity and nitrogen starvation on growth, total fatty acids and arachidonic acid in the green microalga Parietochloris incisa. J. Appl. Phycol. 20, 245-251.

(70) Mendoza, H., Martel, A., Jiménez del Rio, M., and García Reina, G. (1999) Oleic acid is the main fatty acid related with carotenogenesis in Dunaliella salina. J. Appl. Phycol. 11, 15-19.

(71) Rabbani, S., Beyer, P., Lintig, J., Hugueney, P., and Kleinig, H. (1998) Induced beta-carotene synthesis driven by triacylglycerol deposition in the unicellular alga dunaliella bardawil. Plant Physiol. $116,1239-1248$. 